\title{
Enhancing V2X Communication Based on a New Comb-Pilot Estimation Approach
}

\author{
Aymen Sassi, ${ }^{1,2}$ Yassin El Hillali, ${ }^{2}$ Atika Revenq, ${ }^{2}$ Faiza Charfi, ${ }^{1}$ and Lotfi Kamoun ${ }^{1}$ \\ ${ }^{1}$ LETI, ENIS, University of Sfax, Sfax, Tunisia \\ ${ }^{2}$ IEMN, UVHC, Valenciennes, France \\ Correspondence should be addressed to Aymen Sassi; asassi.th@gmail.com
}

Received 30 April 2016; Revised 4 August 2016; Accepted 14 August 2016

Academic Editor: Martin Reisslein

Copyright (C) 2016 Aymen Sassi et al. This is an open access article distributed under the Creative Commons Attribution License, which permits unrestricted use, distribution, and reproduction in any medium, provided the original work is properly cited.

\begin{abstract}
Vehicle to Vehicle (V2V) and Vehicle to Infrastructure (V2I) communication systems, known as V2X technologies, have increasingly attracted attention in current research on road safety and traffic ergonomics. The performance evaluation of these communication systems is an important step before their potential integration and use in real systems. V2X communications are based on the IEEE 802.11p standard also known as Wireless Access in Vehicular Environment (WAVE). V2X can affect human life; therefore a deep study related to V2X performance evaluation should be done in order to be sure about the system reliability. In this context, we have elaborated a deep study related to the effect of transmission range on V2X communications by considering the terminal mobility. First, we have evaluated the performance of the PHY layer on the IEEE 802.11p using simulation. Secondly, we have conducted real case measurements using the Arada LocoMate Transmission system. The obtained results shows the necessity to optimize the quality of transmission in V2X communications. Consequently, we propose in this paper a new comb-pilot technique to enhance the quality of Orthogonal Frequency Division Multiplexing (OFDM) transmission. Our proposal consists in two new uses of the pilot subcarrier estimation technique in order to decrease the elevated bit error rate (BER). The quality of transmission (QoT) is first evaluated relating to the pilot symbol rearranged positions. Second, we proposed to optimize the QoT by adding two supplementary pilot symbols as it can offer better channel estimation results. Based on the performance evaluation of our proposal, it is confirmed that both of rearrangement and the adding of the pilot patterns lead to performance enhancement compared to baseline model (standardized one).
\end{abstract}

\section{Introduction}

The universal demand for mobility and transportation has led to the necessity for the development of practical solutions to lessen the traffic jamming and ameliorate road safety. Since the construction of new roads ceased to be a desirable solution owing to infrastructural and financial concerns, the development of more efficient transportation systems [1] that make use of existing means has emerged as an alternative solution. Thus, a new trend of research concerned with the development of intelligent transportation systems (ITS) [1] arises to support cooperative communication systems [2] incorporating intelligence not only in the vehicles, but also in the surrounding elements in the roadway infrastructure. Consequently, vehicular communication has recently drawn the attention of many researchers all over the world (Japanese,
American, and European [1, 3]) to seek feasible solutions.

Vehicular communication involves two categories; the first one is related to road safety while the second is related to transport ergonomics [4]. Vehicular networks (V2X) (Figure 1) involve two cases, which are Vehicle to Infrastructure (V2I) [5] (Figure 2) and Vehicle to Vehicle (V2V) [5] (Figure 3). V2I covers exchanged data between road infrastructure (such as traffic lights and signs sidewalls) and vehicles. The V2V covers systems that focus on communicating two vehicles without considering an access point control.

It is through Wireless Access in Vehicular Environment (WAVE) or the IEEE 802.11p communication standard that the IEEE community has incessantly worked on the improvement of V2X communication. Actually, it is interested in both the physical PHY layer of specifications for Dedicated Short 


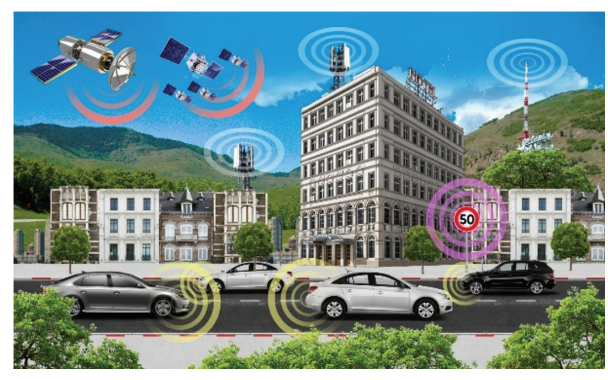

FIGURE 1: Intervehicular communications.

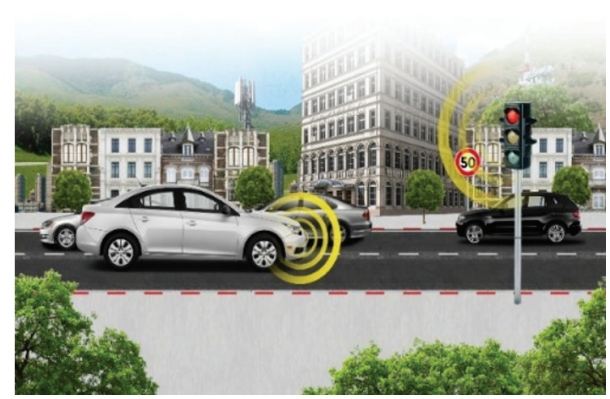

FIGURE 2: Vehicle to Infrastructure communications.

Range Communication (DSRC) and Medium Access Control (MAC) of vehicular communication (Figure 4).

It is noteworthy to mention that the importance of the vehicular communication topic has been underlined by conducting several European projects as illustrated in Table 1. Actually, several testing campaigns have been carried out in a European research project context: iTETRIS, aiming at evaluating the V2I performance by distributing RSU in an urban environment. They came to the conclusion that many parameters, namely, surrounding trees, heavy traffic, and street layout, are among the most important factors that should be considered to optimize transmission. Besides, the range impact on the packet delivery ratio is illustrated by the use of DENSO Wireless Safety Unit (WSU). More interest was focused on measuring the actual IEEE 802.11p performance by NEC Link Bird-MX set on two cars. Furthermore, the results of the WiSafeCar WSC project have been discussed and the intervehicular communication system performance has been estimated in a real-world context. The major findings in this study consist in the high performance of IEEE 802.11p compared to the general performance using traditional Wi-Fi. In the same work, the combination of cellular network (3G) and IEEE 802.11p has been proposed as a hybrid communication technique for upcoming application. Table 1 proposes a highlight of the current main projects that deals with V2X communication systems.

Given the encouraging potentials that this standardized technology may offer for the alleviation of traffic overcrowding and enhancement of road safety, the present research undertakes a new comb-pilot enhancing channel estimation in V2X communication standard.

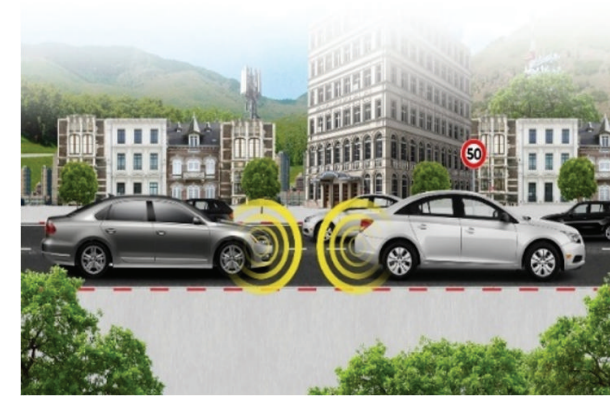

FIgURE 3: Vehicle to Vehicle communication.

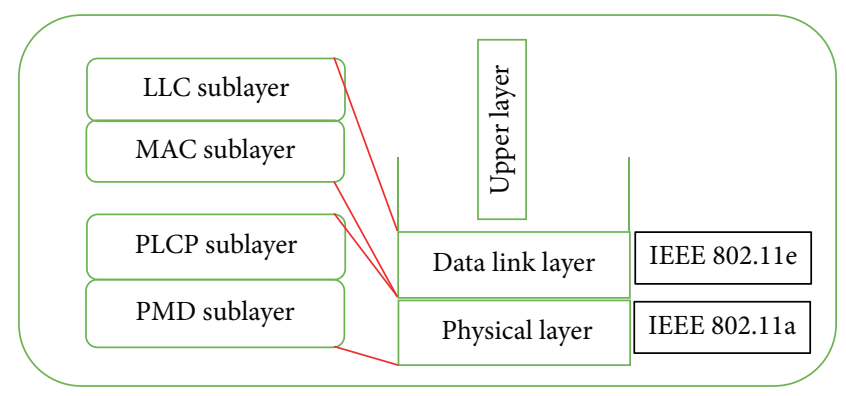

FIGURE 4: IEEE 802.11p layer management.

The main scientific contributions of the paper are focused on the following:

(i) A deep evaluation of the IEEE 802.11p performances.

(a) We started by simulating the analytical transmission model of WAVE communication standard.

(b) We conducted a several sets of real-world experimentations according to different metrics such as communication range and relative speed between nodes.

(ii) Proposition of a solution to overcome the node mobility problem at high data rates, therefore, we focusing on optimizing the channel estimation method by proposing two different base pilot subcarrier channel estimation method.

(a) We begin with rearranging the pilot subcarrier patterns.

(b) Finally, we have proposed two supplementary pilot subcarriers in order to optimize the IEEE 802.11p channel estimation technique.

The rest of the paper is structured as follows. Section 2 presents a review of the literature pertaining to the evaluation of the performance of the IEEE 802.11p communication standard. As for Section 3, it provides a description for the transmission process architecture of this standard, taking operational parameters into consideration. The scenarios and results from the first Matlab-based simulation sets are given in Section 4 which also presents the experimental hardware 
TABLE 1: Comparative international V2X communication projects.

\begin{tabular}{|c|c|c|c|}
\hline Category & Project title & Main goal & Consortium \\
\hline \multirow{3}{*}{$\begin{array}{l}\text { Project based on } \\
\text { cooperative } \\
\text { communication }\end{array}$} & SAFESPOT [23] & $\begin{array}{l}\text { Cooperative system for road safety to prevent road } \\
\text { accidents. }\end{array}$ & $\begin{array}{l}\text { Bosch, Daimler, Volvo, } \\
\text { University of Stuttgart, CNRS, } \\
\text { and so forth }\end{array}$ \\
\hline & DRIVE C2X [24] & $\begin{array}{l}\text { Based on cooperative driving functions and services, it } \\
\text { leads to evaluating their impact on driver behavior. }\end{array}$ & $\begin{array}{l}\text { Audi, Volvo, Renault, Yamaha, } \\
\text { Continental, NEC Europe, } \\
\text { Bosch, IFSTTAR, Raumfahrt } \\
\text { University }\end{array}$ \\
\hline & SCORE@F [25] & $\begin{array}{l}\text { This project deals with developing a cooperative road } \\
\text { traffic system in Europe. }\end{array}$ & $\begin{array}{l}\text { PSA, Renault, HITACHI, } \\
\text { Orange, EURECOM, INRIA, } \\
\text { IFSTTAR }\end{array}$ \\
\hline \multirow{3}{*}{$\begin{array}{l}\text { Safety, accident } \\
\text { assistant \& } \\
\text { incident } \\
\text { management }\end{array}$} & SimTD [26] & $\begin{array}{l}\text { Its objective is to design an electronic brake light } \\
\text { system. This system will allow the vehicle to transmit } \\
\text { first emergency braking message to the following } \\
\text { vehicles on the presence of a potentially dangerous } \\
\text { obstacle. }\end{array}$ & $\begin{array}{l}\text { Audi, BMW, Daimler, Ford, } \\
\text { Opel, Volkswagen, Bosch }\end{array}$ \\
\hline & iTETRIS [27] & $\begin{array}{l}\text { Works with V2X communication technology. It aims to } \\
\text { improve traffic management through real-time data } \\
\text { exchange among V2V or V2I. }\end{array}$ & $\begin{array}{l}\text { Thales, Innovalia Association, } \\
\text { Deutsches Zentrum für Luft, } \\
\text { University Miguel Hernandez de } \\
\text { Elche, Hitachi Europe SAS, and } \\
\text { so forth }\end{array}$ \\
\hline & WiSafeCar [28] & $\begin{array}{l}\text { Its major aim is to implement a reliable wireless traffic } \\
\text { service platform to improve road and traffic safety. }\end{array}$ & $\begin{array}{c}\text { CRP Henri Tudor, Taipale } \\
\text { Telematics, Sunit, Ubridge, FMI, } \\
\text { VTT }\end{array}$ \\
\hline
\end{tabular}

and scenarios conducted, as well as the obtained results and conclusions. As for Section 6, it closes the present research work with a summary and future perspectives.

\section{Related Works}

The area of intelligent transportation systems (ITS) has been the interest of many research works $[7,8]$ leading to the enhancement of the transport quality considering the existing public infrastructure. Indeed, many research works have been interested in the performances of these communication systems. Accordingly, in this section, we overview V2X communication related work by considering respectively contributions focused on MAC layer $[9,10]$, PHY layer performance through analytical evaluation $[3-5,7,8]$, real-world experimentations [10-12], and enhancing WAVE proposals [13-15]. A brief summary of the different works is described in Table 2.

Authors in $[9,10]$ have investigated their work on simulating and evaluating the MAC and upper layer performance of the IEEE 802.11p standard. Eichler [9] provided a performance evaluation of the IEEE 802.11p standard by considering three metrics: the delay, throughput, and collision probability. Their study was conducted using analytical and simulation means. The main results of this work is the fact that the standard IEEE 802.11p is not able to support heavy leads. In this case, the throughput decreases significantly while the delay increases.

In another study, Wang et al. [10] have also investigated their work on simulating the IEEE 802.11p MAC protocol for the V2I communication. The NS-2 simulation results showed that the used protocol can achieve a poor throughput performance due to the backoff time size. In order to overcome this problem, the authors have proposed two different approaches for improving the MAC protocol under dense and dynamic conditions. The first one is a centralized approach that has the capacity to calculate the best size of the backoff window according to the exact number of transmitting vehicles. Concerning the second approach, it is a distributed one in which vehicles are capable of using indigenous observations to adjust the window size.

Other research works have been interested in the study of the performance of the WAVE standard according to a certain number of parameters such as throughput, delay, packet size, mobility impact, and urban environment. In fact, Jafari et al. [11] have analyzed the IEEE 802.11p standard and implemented a set of measures in an NS-2 network simulator using a realistic model based on vehicular mobility. In their simulation scenarios, they have focused on the throughput, end-to-end delay, and Packet Loss Ratio (PLR) to highlight the effect of mobility and packet size on the WAVE standard. Their results revealed that the likelihood of effective message reception is the same when the distance is less than 138 meters for all vehicles used. They have also led to the conclusion that the average of the throughput and the end-to-end delay metrics increased with the increase in the message sizes. Furthermore, Park et al. [12] have studied the impact of the limited packet size on vehicular networks. To solve this constraint, the authors built their analysis on the transmission rate according to a different data set as well as on length size to optimize the IEEE 802.11p transmission quality. Both Sassi et al. [13] and Demmel et al. [14] tried a measurement field of 802.11p communication technology on track experimentation. Maximum range, packet loss rate, 


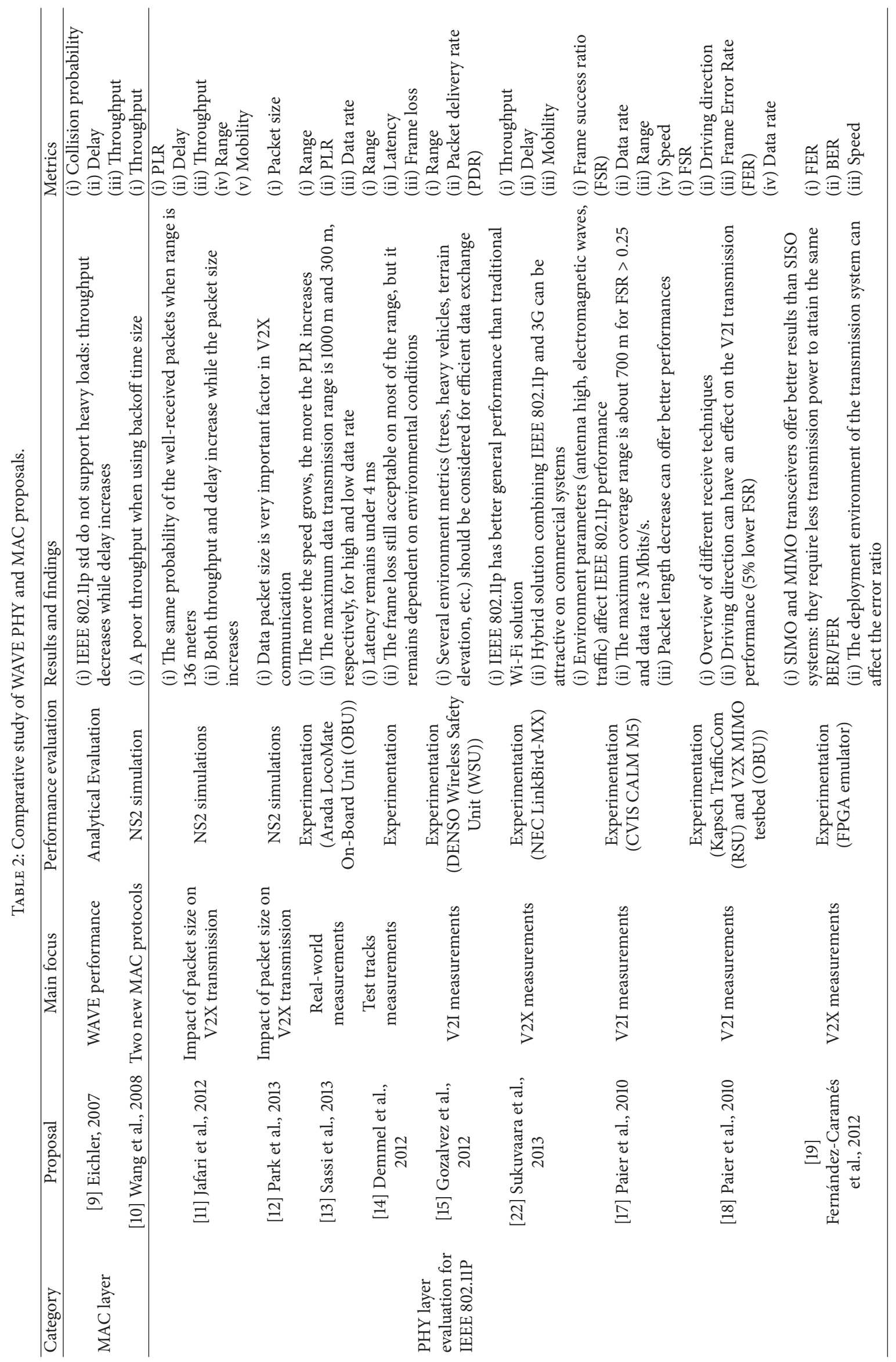




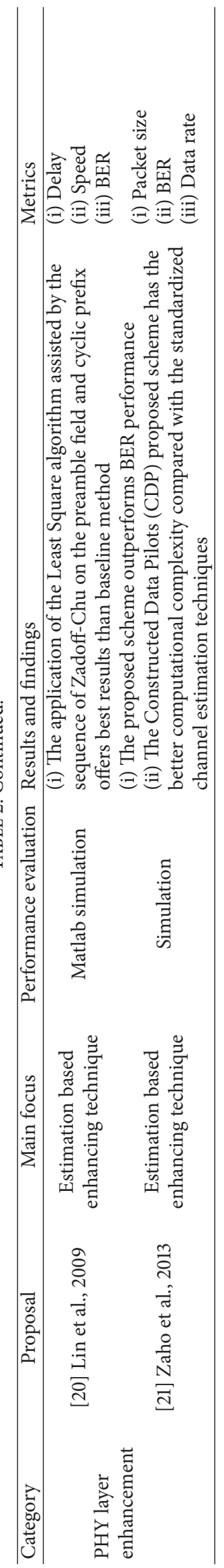


and other speed impacts are some of the metrics elaborated on in these works. Their results have proven that the more the range grows, the worse the transmission quality will be. They have also shown that the more the speed increases up to $50 \mathrm{~km} / \mathrm{h}$, the more the PLR of all tested modulations worsen. Gozalvez et al. [15] have explored the future exploitation of V2X communication in urban environments. They have conducted a set of IEEE 802.11p V2I-based measurements and highlighted the impact of urban characteristics and infrastructure on the IEEE $802.11 \mathrm{p}$ transmission quality. Their results have shown that several parameters, including the presence of heavy vehicles, traffic density, and field altitude, should be considered for optimal transmission.

An overview of the literature shows that the supply of vehicles with smart technologies allowing the efficient communication with surrounding settings is crucial to the progress of next intelligent transportation systems generation. It has been reported that the performance efficacy of this communication systems depends on various parameters, namely, environmental and mobility factors. Although there has been a current development in the WAVE field and exceptional breakthroughs in ITS technology, the need still exists for studies to further investigate the information transmission and reception between vehicles and their real environments. In order to have an idea about the real performance of the intervehicular communications, so many experimental studies have been carried out. In this regard, Paier et al. [17] reported on an outdoor V2I trial based on the IEEE 802.11p PHY protocol. Their measurement results on highway presented the average downstream performance. The authors have concluded that the vehicles shadowing effects may cause a fluctuating performance, especially when using a long-size packet with high vehicle mobility. The studies of Paier et al. [18] have been oriented to the assessment of the PHY layer in the standard of V2I communication. Indeed, a series of real-life measurements along a highway has been performed and various scenarios involving several packet lengths, data rates, and vehicle speeds have been considered. They have confirmed that the fixed antenna height may influence the transmission quality. The authors have assessed the performance of communication with an OBU speed of $120 \mathrm{~km} / \mathrm{h}$ and come to the conclusion that speed may completely decline transmission. Actually, their results elucidate the worsening of the rate of the frame error to 0.1. In another study, Maier et al. [6] presented the performance evaluation results of a multiantenna receiver based on the IEEE 802.11p communication standard. The evaluation involved real-world measurements related to the Selection Combining (SC), Equal Gain Combining (EGC), and Maximum Ratio Combining (MRC) algorithms. The obtained results showed that, despite poor receiving conditions, reliability and robustness can be significantly improved. In fact, they have reported that the frame success ratio was increased by up to $25 \%$. The focus of the work of Maier et al. in [6] was on the assessment of IEEE 802.11p performance. By means of Kapsch TrafficCom equipment, their experimentations were interested in V2I communication. Their analysis was realized by three linking techniques: the Selection Combining (SC), the Maximum Ration Combining (MRC), and the Equal Gain Combining
(EGC). Their most important results pertain to the influence of the message length on the frame error ratio. Actually, they came to the conclusion that large frames bring about considerably increased error probabilities.

In order to overcome the damage applied to transmissions in intervehicular communications, several studies have been developed to improve their performance.

Improving the IEEE 802.11p communication performance may also be achieved through the use of multiple antennas. Indeed, Fernández-Caramés et al. [19] described the design and implementation of such technique based on two IEEE 802.11p software transceivers and two FPGA channel emulators. They have proved that WAVE communication can be considerably enhanced by using the MIMO system. They reported that performance evaluation could be accelerated from 6 to 209 times as compared to the standard. Lin et al. [20] have proposed a method based on time domain estimation to pass by the insufficient bandwidth coherence for channel estimation according to a rich scattering. The enhancement found in their work was obtained through the application of the Least Square algorithm assisted by the sequence of Zadoff-Chu on the preamble field and cyclic prefix. The work of Zhao et al. [21] focused on the analysis of channel estimation schemes dedicated for the intervehicular communication systems. The authors proposed a novel channel estimation scheme based on pilot symbols. Their technique consisted in changing data symbols into pilot ones. The results of their analysis and simulation have shown that the suggested estimation is likely to enhance the standardized scheme in high SNR. Last but not least, Sukuvaara et al. [22] have based their research on experimenting the intervehicular communication performances. They have paired both IEEE 802.11p standard and 3G cellular network for test measurements. They tried to study an intelligent traffic safety system using a V2I communication architecture. Based on both measurement fields and preliminary deployment, they have suggested a genuine system deployment strategy for simple scenarios. They have demonstrated that the use of pilot system can properly provide defined services and concluded that the deployment of such hybrid method could lead to a promising solution for the vital commercial system.

To offer a better improvement for intervehicular communications, a comprehensive performance evaluation is required. The overview of the previous studies shows that they were either experimental or theoretical, but no study has been interested in the comparison between them. The research works that have proposed techniques to improve performance WAVE were not based on this real case situation. Therefore, we propose a V2X performance survey based on IEEE 802.11p comparison between real-world experimental measurements and theoretical results. Based on this results compilation, we will propose a novel channel estimation technique in order to enhance the performance of its performance.

\section{IEEE 802.1p PHY Layer Transmission Model}

The physical layer of the IEEE 802.11p is similar to that of the IEEE 802.11a. It includes two sublayers. The first sublayer, 


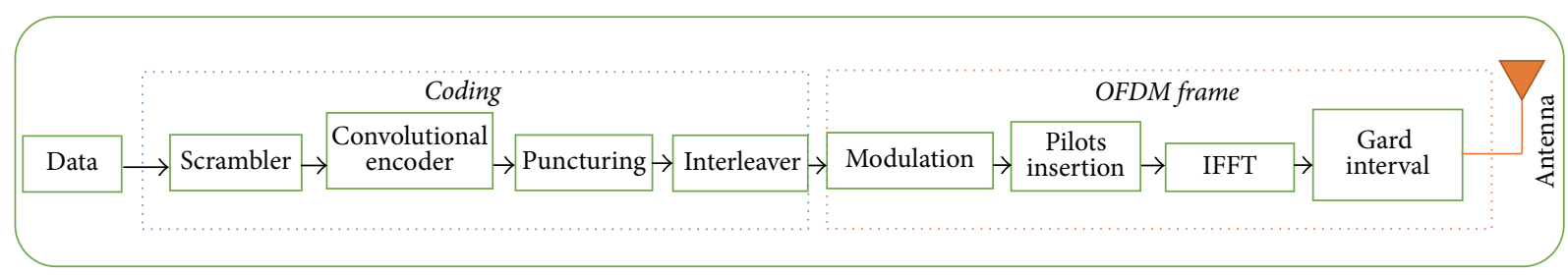

FIGURE 5: IEEE 802.11p transmission model.

Physical Layer Convergence Protocol (PLCP), assures communication with the upper layer MAC. It transfers the Packet Data Unit (PDU) received from the MAC upper layer into an OFDM frame. The second sublayer Physical Medium Access (PMD) acts as an interface with physical transmission medium. It provides data encoding and modulation. Therefore, the IEEE 802.11p schema involves two sets submodules related to coding and OFDM frame content as illustrated in Figure 5.

Accordingly, the IEEE 802.11p transmission procedure consists of numerous steps (scrambler, convolutional encoder, puncturing, interleaver, modulation, pilot insertion, IFFT, and guard interval (GI)). An exhaustive description of this transmission procedure is given in the following related works $[29,30]$.

In our work, we have considered the following physical layer parameters.

To generate required data, a data source component was used to reduce the data unit that should be processed by the physical layer.

To avoid the presence of long bit sequences that may lead to transmission errors, a scrambler should be used. The scrambler component considered in this work brings about a 127-bit sequence based on the following function:

$$
S(x)=x^{7}+x^{4}+1
$$

A convolutional encoder is employed, at a $1 / 2$ coding rate, to circumvent possible undesirable effects induced by the Intersymbol Interference (ISI) and Intercarrier Interference (ICI) on the produced data and to identify and correct errors. It is also used to guarantee the redundancy addition to the transmitted bit stream.

The output of convolutional encoder is forwarded to a puncturing element to produce upper $R=3 / 4$ and $R=$ $2 / 3$ coding rates. The decrease of the number of bits to be transmitted by puncturing leads to the coding rate increase. It requires some of the coded bits omission from the transmitter side and their replacement by "zeros" in the convolutional decoder on the receiving side. The puncture model is identified by the binary puncturing vector corresponding to two-bit sequences: 1110 for rate $R=2 / 3$ and 110101 for rate $R=3 / 4$.

The coded data are incorporated to circumvent error bursts induced by channel fading. The interleaving process is made up of a two-step permutation in time and frequency domains. Concerning the first permutation, it attempts to guarantee that there are not two consecutive bits which are coded in two contiguous subcarriers. As for the second permutation, it assures that two subsequent bits are interchangeably represented in the most and least momentous bits of the constellation employed.

The data modulation is conducted by the phase shift keying (BPSK or QPSK) or amplitude modulation (16-QAM or 64-QAM). The data throughput is affected by the selection of the mapping and coding rate, ranging between $3 \mathrm{Mb} / \mathrm{s}$ (with BPSK and $1 / 2$ coding rate) and $27 \mathrm{Mb} / \mathrm{s}$ (with 64QAM and 3/4 coding rate). Furthermore, the bit streams are transformed into symbols for simultaneous transmission. Hence, the aim of using the OFDM technique is to convert the sequential data stream into parallel streams and that of the Inverse Fourier Transform (IFT) technique is to modulate those data onto orthogonal subcarriers. The total number of existing subcarriers is 64 , among which only 52 information carriers are used for mapping. In fact, the application of the 11 guard subcarriers on the OFDM spectrum sides aims to separate them from adjacent subbands. This step involves the positioning of the intricate symbols that are associated with various constellation points on subcarriers. The 52 subcarriers used in the IEEE 802.11p were composed of 48 data subcarriers and 4 pilot subcarriers. The aim of using the pilot subcarriers is to ensure the detection robustness against frequency offsets and phase noise, whereas that of using the pilot symbols is to detect the channel and note the changes introduced to the transmitted signal. The pilot subcarriers are interleaved in subcarriers $-21,-7,21$ and 7 . From the frequency domain, the OFDM symbols are converted to the temporal domain using IFFT in order to convey the data on subcarriers.

Before each OFDM symbol, a second guard interval (GI) is introduced to avoid the occurrence of ISI and ICI problems due to multipath propagation. Besides, the IEEE 802.11p (Draft 9.0) [29] defines the duration of $\mathrm{GI}_{1}$ as being equal to $\mathrm{TGI}_{1}=\mathrm{TFFT} / 4$ and lies in the copying of the end of the OFDM symbol in the start of the symbol that follows.

\section{IEEE 802.11p Performance Evaluation}

4.1. Scenarios. In this study, the used scenarios focused on both simulation and real-world experiments. They involved two complementary fields, that is, the IEEE 802.11p real-world performance and the dedicated WAVE model consistency, where the Matlab simulator and experiments were taken into consideration.

4.1.1. Matlab Simulation Scenarios. This section describes multiple simulations scenarios aiming to evaluate the performance of intervehicle communication. The carried 
TABLE 3: Simulation parameters.

\begin{tabular}{|c|c|c|}
\hline Parameters & \multicolumn{2}{|c|}{ Values } \\
\hline Frame symbols & \multicolumn{2}{|c|}{1000} \\
\hline Number trials/scenario & \multicolumn{2}{|c|}{100} \\
\hline \multirow{2}{*}{ Mobility } & \multirow{2}{*}{$\begin{array}{l}\text { Two cases are } \\
\text { considered }\end{array}$} & Moving vehicles \\
\hline & & Stopped vehicles \\
\hline Data rate & \multicolumn{2}{|c|}{$3,4.5,6,9,12,18,24,27 \mathrm{Mbits} / \mathrm{s}$} \\
\hline \multirow{4}{*}{ Modulations } & \multicolumn{2}{|c|}{$\operatorname{BPSK}(1 / 2,3 / 4)$} \\
\hline & \multicolumn{2}{|c|}{ QPSK $(1 / 2,3 / 4)$} \\
\hline & \multicolumn{2}{|c|}{16 QAM $(1 / 2,3 / 4)$} \\
\hline & \multicolumn{2}{|c|}{64 QAM $(2 / 3,3 / 4)$} \\
\hline Transmission channel & \multicolumn{2}{|c|}{ Rice (to simulate urban environment) } \\
\hline Doppler shift & \multicolumn{2}{|c|}{ Considered } \\
\hline \multirow{3}{*}{ Speed } & \multicolumn{2}{|c|}{ 1st scenario $($ speed $=0 \mathrm{~km} / \mathrm{h})$} \\
\hline & \multicolumn{2}{|c|}{$\begin{array}{l}\text { 2nd scenario (speed from } 0 \text { to } \\
\qquad 50 \mathrm{~km} / \mathrm{h})\end{array}$} \\
\hline & \multicolumn{2}{|c|}{$3 \mathrm{rd}$ scenario (speed up to $260 \mathrm{~km} / \mathrm{h}$ ) } \\
\hline
\end{tabular}

simulation aimed to examine the QoT according to essentially the SNR effect.

Two case studies were considered: one corresponds to the V2V while the other deals with the V2I communication. The same number of symbols in a transmitted frame $(1000$ symbols) is used in the 100 iterations for both scenario cases. The parameters deployed for simulation are regrouped in Table $3[30]$.

4.1.2. Real-World Experimentation Scenarios. The conducted scenarios were built on real-world experimental testing, using Arada Systems LocoMate OBU, a multimodal communication device compliant with the IEEE 802.11p communication standard. The Arada Systems LocoMate is a leading developer of technologies for communication network applications based on vehicles, including services of vehicle safety, tools collection, and commerce dealings through vehicles. Regarding LocoMate, it has been thoroughly assessed for instantaneous communication between vehicles and roadside access points or other vehicles, thus producing a real-time network of public safety [19]. Furthermore, the Arada Systems LocoMate OBU device may offer wireless communication in a vehicular environment while taking various data rates relatively to the IEEE 802.11 p standard into consideration. Using this standard, low-potential connectivity is provided for intervehicle and vehicle to roadside units. Such a solution incorporates a GPS device to vehicle navigation. As can be seen from Figure 6, the used $5.9 \mathrm{GHz}$ antennas in our experiment and the Arada LocoMate OBU are presented.

Figure 7 shows that the experimental scenarios of this study were performed on a $1.5 \mathrm{~km}$ long landing runway of an unexploited Cambrai airstrip. The tests involved the use of two cars supplied with devices of Arada LocoMate OBU. The tests concentrated on the effects of receiving signal quality, Received Signal Strength Indication (RSSI), distance between the sender and the receiver, data rate, and vehicle speed. Packets were dumped from a monitor interface that was

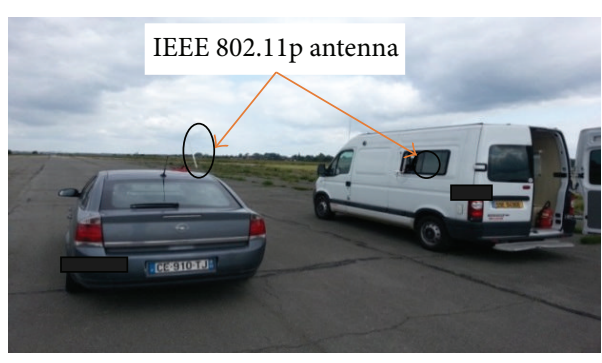

FIGURE 6: Landing runway of an unused Cambrai airfield.

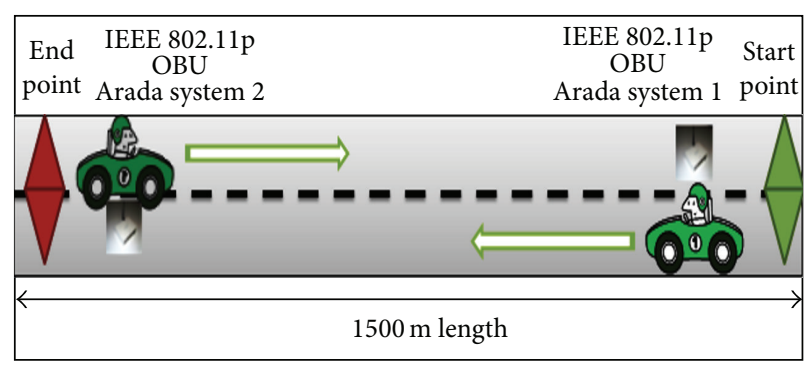

FIGURE 7: Fixed scenarios area.

capable of obtaining different MAC layer parameters on the sender, including the results of the of the Packet Lost Ratio (PLR) and RSSI measurement. The results were collected at $10000 \mu$ second intervals between two consecutive packets. We have considered the same number of transmitted symbols and the same data rates as well as those applied in the simulation carried with Matlab tools. The number of trials was set to 10 per each scenario.

4.2. Results and Discussions. The obtained simulation and experimental results can be gathered into three areas of interest. Concerning the first one, it pertains to the effect of the signal to noise ratio on transmission quality. The second set concerns the influence of moderate speed mobility on V2I communication performance. As for the third cluster of results, it relates to the high speed mobility impact on V2V communication.

4.2.1. Agreement between Simulation and Experimentation Data in relation to SNR Effects on Transmission Quality. The first area of interest is about the consistency of SNR effect on the QoT of both simulations and experiments. Simulations were performed according to the eight possible modulations

$$
\mathrm{SNR}=\frac{P_{r}}{P_{\text {noise }}},
$$

where $P_{r}$ aims to signal power while $P_{\text {noise }}$ aims to noise power. Aiming at representing the relation between the SNR (2) and the distance between sender and receiver, we have used the Friis transmission equation:

$$
\frac{P_{r}}{P_{t}}=G_{t} * G_{r} *\left(\frac{\alpha}{4 \pi D}\right)^{2},
$$

where $G_{t}$ and $G_{r}$ refer to the transmission antenna and reception antenna gain, respectively, $D$ refers to the range 
between the sender and receiver, and $\alpha$ refers to the wave length as given in

$$
\alpha=\frac{v}{f},
$$

where $v$ refers to the phase velocity magnitude and $f$ to wave frequency.

Our set of experimentations leads to the conclusion that only the $D$ range would change. We can conclude that the more the $D$ range increases, the more the SNR value decreases, as illustrated

$$
\mathrm{SNR}=\frac{P_{t} * \varphi}{P_{\text {noise }} * D^{2}}
$$

The reason behind conducting the first field experiment tests was the evaluation of the range of maximum connectivity of the IEEE 802.11p. In this case, the distance between the two vehicles (where one acts like transmitter and the second acts like a receiver) is a variable ranging from 100 to 1000 meters.

As revealed from the first scenario, the range theoretical maximum of $1000 \mathrm{~m}$ in the IEEE $802.11 \mathrm{p}$ standard was proven only in low data rates of 3 and $4.5 \mathrm{Mbits} / \mathrm{s}$. Yet, a high rate of data is likely to be used $(18,24$, and $27 \mathrm{Mbits} / \mathrm{s})$ in the case of close distance between the vehicles (less than $200 \mathrm{~m}$ ). Such results may be accredited to the quality of the received signal which was found to improve with the stability of the transmission power and the increase in the range of distance communication. These experiment results also postulate that PLR values worsened with the increase in the data rate, reaching $0 \%, 1.8 \%$, and $45 \%$ with 3,12 , and $27 \mathrm{Mbits} / \mathrm{s}$, respectively, for the $200 \mathrm{~m}$ range. Consequently, the decrease of the range presented in Figure 8 corresponds to the increase of the SNR shown in Figure 9.

In brief, the achievement of connection efficiency, particularly if the sender's car is distant from the receiver vehicle (more than $200 \mathrm{~m}$ ), can be said to necessitate the application of a low data rate. By compiling all curves in plots, the QoT is shown to increase with better SNR levels (the range decreases).

4.2.2. Agreement between Simulation and Experimentation Data with regard to the Mobility Impact of on V2I. The second simulation series aimed to study the V2X communication by considering an urban environment where the relative speed cannot exceed $120 \mathrm{~km} / \mathrm{h}$. The mobility is thus considered as an important factor in V2X communication. It is to be noted that the speed increases (from 50 to $90 \mathrm{~km} / \mathrm{h}$ ) with the increase of BER (from $2.310^{-2}$ to $9.510^{-2}$ ) for OFDM modulation BPSK $1 / 2$.

The second experiments set aimed at evaluating the real V2I communication performance. Actually, an Access Router (AR, roadside unit) of the sender was represented by a first stopping vehicle and a mobile router ( $M R$, vehicle) of the receiver using many speeds in the range of 10 and $110 \mathrm{~km} / \mathrm{h}$ was epitomized by a second one. Figure 5 reveals that the same starting point for the MR was fixed to retain the same measurement conditions in all iterations.

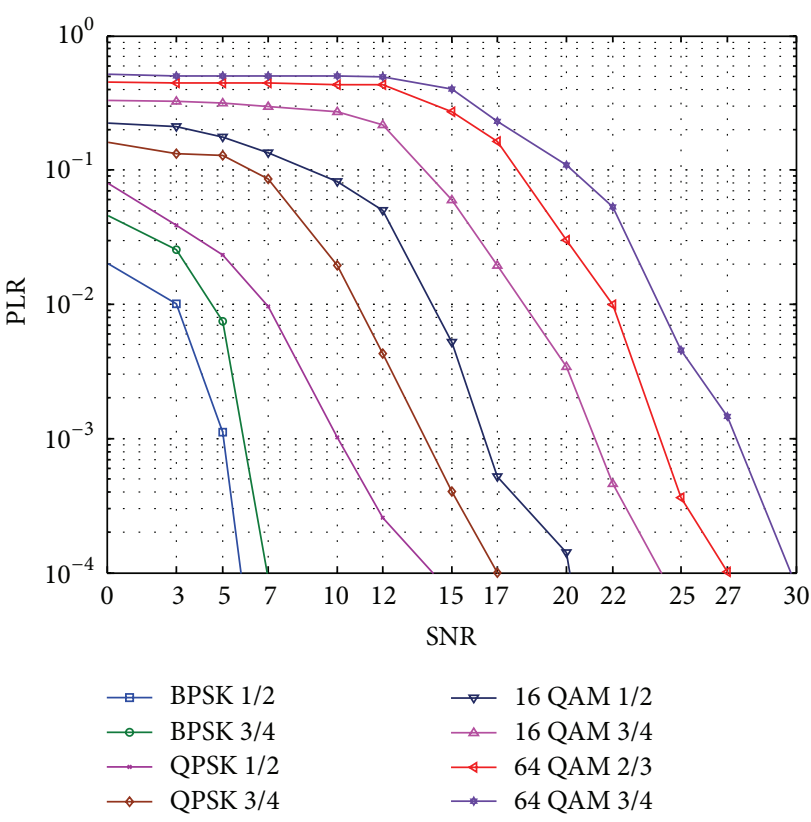

FIGURE 8: Impact of SNR on the BER for different modulations with Matlab simulation [6].

The findings from this comparative analysis confirm that the curve shapes generated in our Matlab-based simulation are relatively in good agreement with the evolution of the curves modeled in the experimental system. Figures 10 and 11 present the effect formerly seen for the rise of the data rate on the PLR, taking into account different speeds in this scenario.

The relationship between the PLR and the V2I communication speed variation is displayed in Figure 10. The curves in Figure 11 show that the PLR worsens with the increase of the speed level until reaching a sound sight at $110 \mathrm{~km} / \mathrm{h}$, which can apparently be accredited to the Doppler shift.

\subsubsection{Agreement between Simulation and Experimentation} Results with regard to the Mobility Impact on $V 2 V$. In the third simulation scenario, the attempt was to evaluate V2X communication for the different modulations while taking the relatively high speed (from 10 to $260 \mathrm{~km} / \mathrm{h}$ ) into account. It can be clearly seen that high mobility exerts negative effects on the QoT. Actually, as obviously noted in Figure 12, the findings show that, with speeds reaching $190 \mathrm{~km} / \mathrm{h}$, the eight modulation curves become gradually close to each other according to speed growth. Nevertheless, the BPSK $1 / 2$ seems to be the best modulation type.

The third scenario with maximum speed of $220 \mathrm{~km} / \mathrm{h}$ was undertaken to assess the actual performance of WAVE in the $\mathrm{V} 2 \mathrm{~V}$ condition. Indeed, the relative speed concept was used, with both vehicles moving in reverse directions at a speed range of 10 and $110 \mathrm{~km} / \mathrm{h}$, so as to get a speed in the range of $20-220 \mathrm{~km} / \mathrm{h}$. As in the V2I scenario and as demonstrated in Figure 5, the same starting transmission point was restricted in this experiment for both vehicles. So, at the reception unit, the packet reception performance was obtained.

Figure 12 shows the effect of the high speed range as a function of the PLR on V2V communication. Such results 


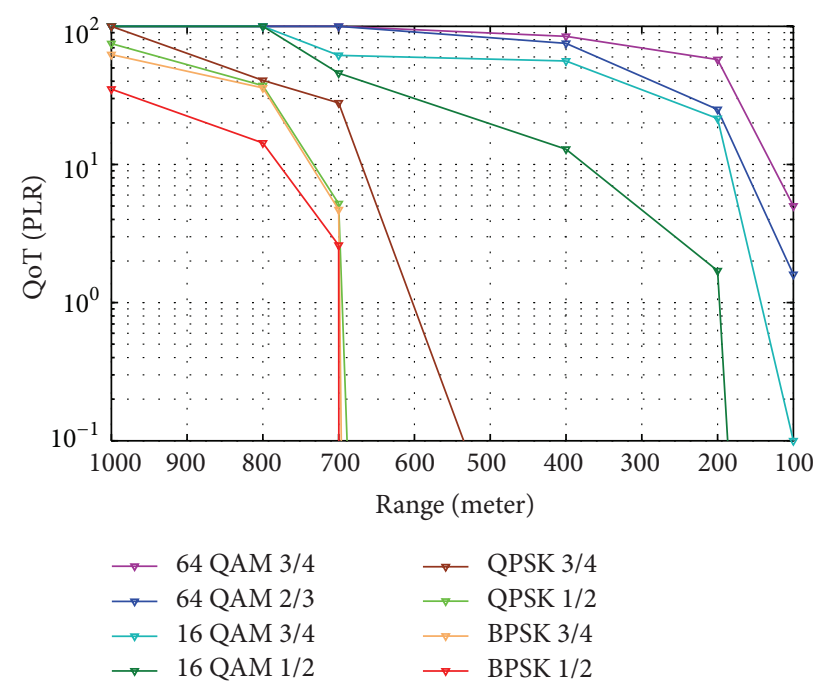

FIGURE 9: Impact of range on the PLR for different modulations with real-world experimentation.

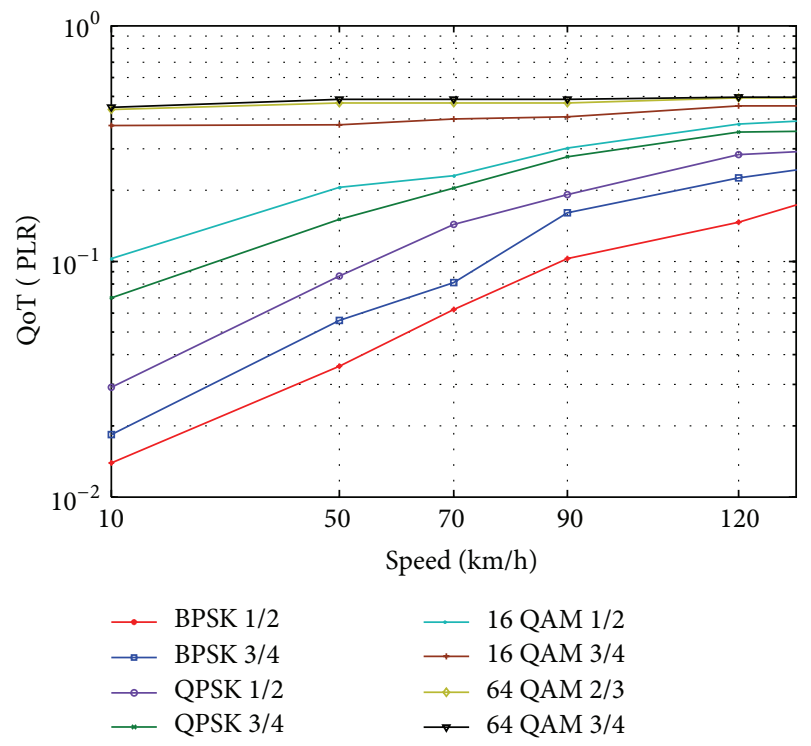

FIGURE 10: Mobility impact on V2I with Matlab simulation (speed 20 to $120 \mathrm{~km} / \mathrm{h}$ ) [16].

offer remarkable feasible insights that can be summarized as follows. Indeed, the application of low data rates prompts more important effects of node mobility on vehicular communication than higher data rates. According to the results presented in Figures 12 and 13, an increase from 5\% to $11.5 \%$ in PLR can be obtained for the data rates of 3 and $27 \mathrm{Mbits} / \mathrm{s}$ at $50 \mathrm{~km} / \mathrm{h}$, respectively. Eventually, the quality of transmission degrades with the increase of the node speed; that is, the PLR increases from $6 \%$ to $18 \%$ for $40 \mathrm{~km} / \mathrm{s}$ and $220 \mathrm{~km} / \mathrm{h}$, respectively, for the BPSK $1 / 2$. Therefore, both figures (Figures 12 and 13) agree well with their curves shapes.

With respect to the long-range communications, it is obvious that using low data rate is favored for more enhanced performances. Besides, the communication can be affected

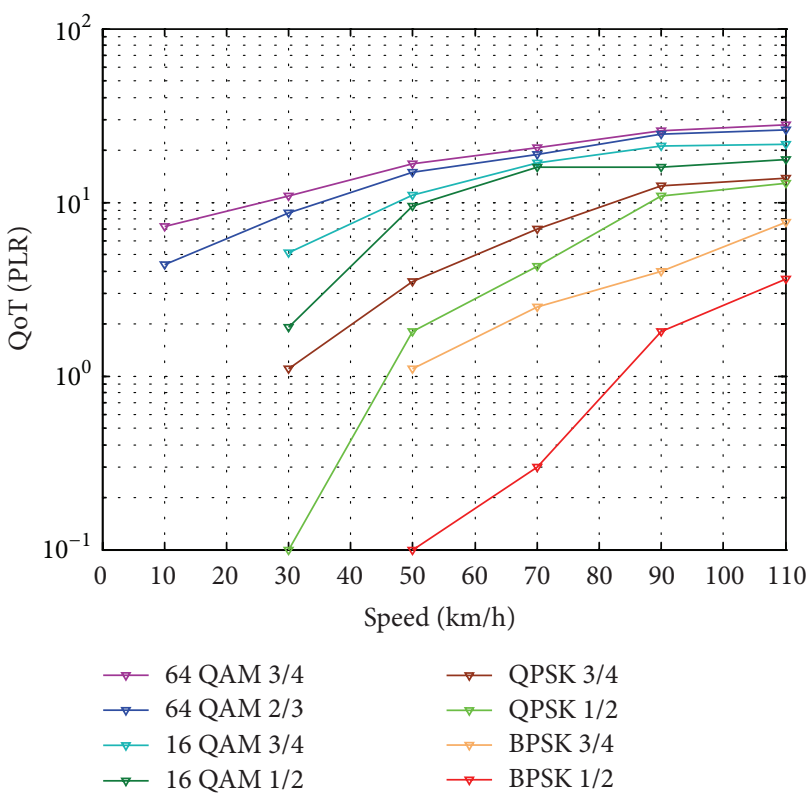

FIGURE 11: Mobility impact on V2I communication with real-world experimentation (speed from 20 to $110 \mathrm{~km} / \mathrm{h}$ ).

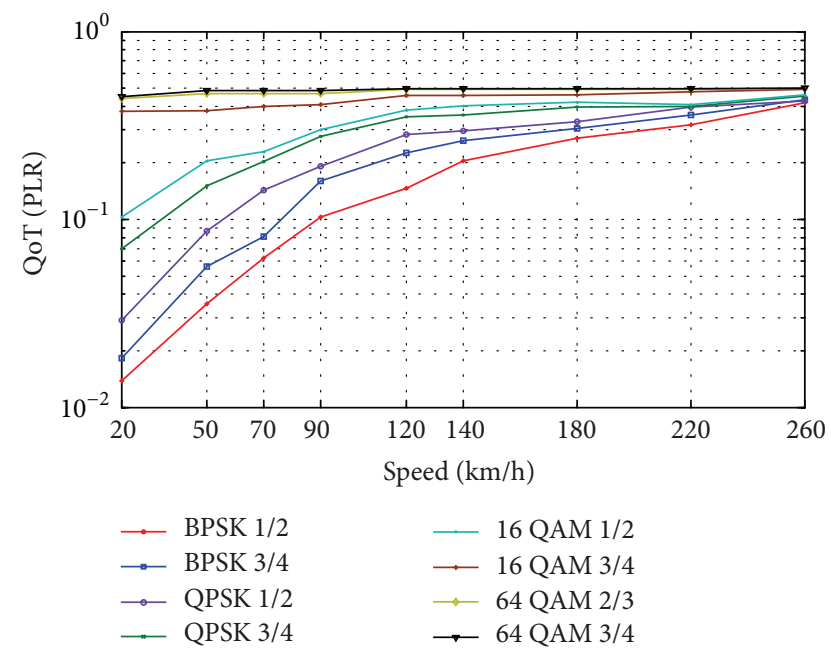

FIGURE 12: Mobility impact on V2V communication with Matlab simulation (speed from 20 to $260 \mathrm{~km} / \mathrm{h}$ ) [6].

negatively by node mobility. As for V2V communication, the transmission quality gradually decreases with the increase of the node speed gradually. In the following section, a new channel estimation technique based on the comb pilots is proposed in order to overcome this loss.

\section{V2X Communication Optimizations Based on Comb-Pilot Estimation Approach}

The fundamental of the suggested approach in the present research work is an estimation of the channel with pilot symbols. Actually, two scenarios series are realized. In the first one, the impact of the rearranged position of the pilot symbol on the transmission quality QoT [13] is highlighted, and in 


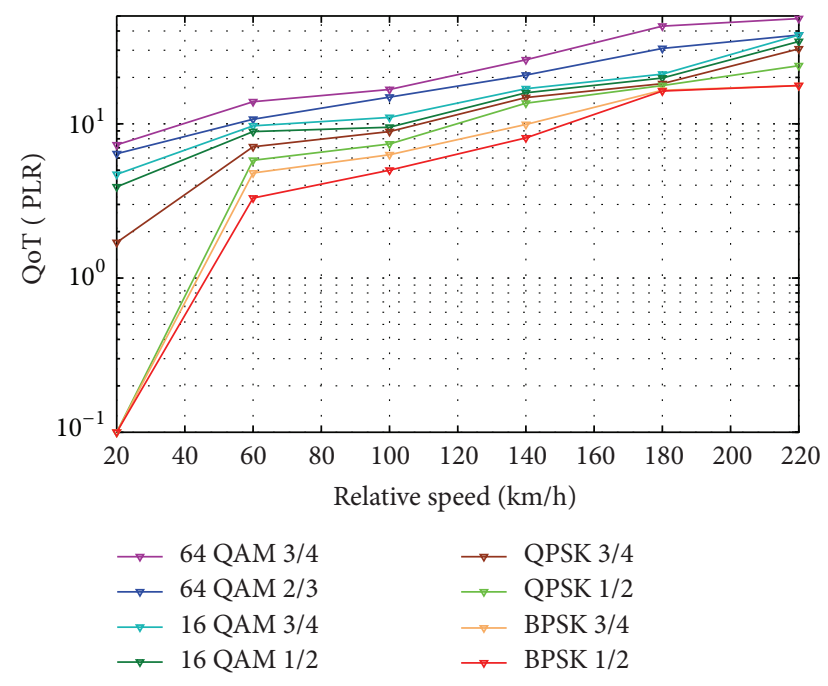

FIGURE 13: Mobility impact on V2V communication with real-world experimentation (speed from 20 to $220 \mathrm{~km} / \mathrm{h}$ ).

TABLE 4: Enhancing proposition parameters.

\begin{tabular}{|c|c|c|}
\hline Parameters & \multicolumn{2}{|c|}{ Values } \\
\hline Frame symbols & \multicolumn{2}{|c|}{1000} \\
\hline Number trials/scenario & \multicolumn{2}{|c|}{1000} \\
\hline Mobility & $\begin{array}{c}\text { Two cases are } \\
\text { considered }\end{array}$ & $\begin{array}{c}\text { Moving vehicles } \\
\text { at } 50 \mathrm{~km} / \mathrm{H}\end{array}$ \\
\hline Data rate & \multicolumn{2}{|c|}{$3,4.5,6,9,12,18,24,27 \mathrm{Mbits} / \mathrm{s}$} \\
\hline Modulations & \multicolumn{2}{|c|}{$\begin{array}{c}\text { QPSK }(1 / 2,3 / 4) \\
16 \operatorname{QAM}(1 / 2,3 / 4) \\
64 \operatorname{QAM}(2 / 3,3 / 4) \\
\end{array}$} \\
\hline Transmission channel & \multicolumn{2}{|c|}{ AWGN } \\
\hline
\end{tabular}

(1) Standard pilot positions

Scenario

(2) Rearranged pilot positions

(3) Two added pilot positions

the second, the impact of two new pilot symbols addition on the transmission process is illustrated. In order to ensure an appreciated level of confidence results, the trials of the proposed scenarios were fixed to 1000 iterations. The main parameters of the carried out simulation are summarized in Table 4.

5.1. Optimized Pilot Position Method. At this level, a variation of BER has been performed according to SNR with a special focus on the influence of the pilot symbol positions change in eight types of modulation. Besides, the scenario specifications takes into account the transmission of 1000 symbols on an AWGN channel model with a fixed vehicle speed at $V=$ $50 \mathrm{~km} / \mathrm{h}$. Regarding the primary case, the channel estimation was set in function of an uneven spacing between the schema of the subcarriers. The spacing positions $[-21,-7,7,21]$ between the pilot subcarriers have an influence on the nonestimated subcarriers number (maximum 13 in Figure 14).
Aiming at the enhancement of the performance of the transmission, the reduction of these nonestimated subcarriers number was proposed. The pilot subcarriers were relocated at $[-18,-9,9,18]$ position with an even spacing pattern of eight, as shown in Figure 14. This proposed technique is beneficial in terms of the reduction of the nonestimated subcarriers number to eight.

According to Figures 15, 16, and 17, using the technique of the suggested rearranged pilot position, the pilot subcarriers can be evidenced to offer better simulation results by the reduction of the nonestimated subcarriers series. The channel state is proven to be more predictable and therefore boosts the bits transmission.

5.2. Optimized Pilots Number Method. In order to study the two scenario sets, a comparison between the suggested and standardized pilot positions was made, and then two supplemented pilot subcarriers were proposed to be appended.

Given the used subcarrier number being 52 and the used IFFT elements offering 64 subcarriers, allocating 2 new subcarriers to pilot symbols from the 8 nonused ones is suggested [30]. The total number of pilot subcarriers is then raised from 4 to 6 shown in Figure 18.

For these simulations, similar number of bits to be conveyed was used (1000 symbols). The transmission speed will be $V=50 \mathrm{~km} / \mathrm{h}$, taking into account the AWGN channel model for the eight types of modulation with the method of Minimum Mean Square Error (MMSE) estimation.

The blue curves, in Figures 19, 20, and 21, illustrate the bit error rate variation according to SNR that takes place with the addition of two pilot subcarriers. With the accumulation of all plots, the description of the rearranged pilot subcarrier effect shown in the green curves can be confirmed to yield better results than the standardized positions. The proposed approach was then found to offer the highest values over all cases. The comparison between Figure 15 and Figures 20 and 21 shows that the obtained results become better with the increase in the data rate. Despite this improvement, the addition of pilot subcarriers will have an impact on the spectral efficiency (SEF). The latter is defined as the ratio between the pilot subcarrier and the total number of used subcarriers, increasing from 0.076 to 0.115 .

\section{Conclusion}

The present research work aimed to design a set of Matlabbased simulations for $\mathrm{V} 2 \mathrm{X}$ communication relative to the IEEE 802.11p communication standard and a supplementary set of real-world experimentations using the Arada Systems LocoMate OBU multimodal communication device. The correlations between the findings from the two sets of evaluation scenarios with regard to the performance of the IEEE 802.11p communication standard were examined. The obtained results have confirmed that using a low rate of data is favored for more enhanced performances in the long-range communications. The findings have also shown that communications can be affected negatively by node mobility. Moreover, in the case of V2V communication, the experimental data indicate that the quality of transmission 


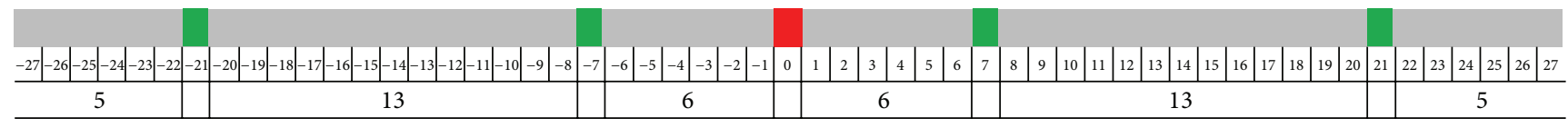

Standard position

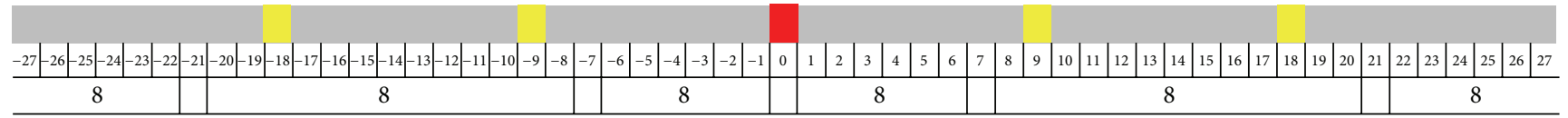

Proposed position

FIGURE 14: Rearranged pilot subcarrier positions.

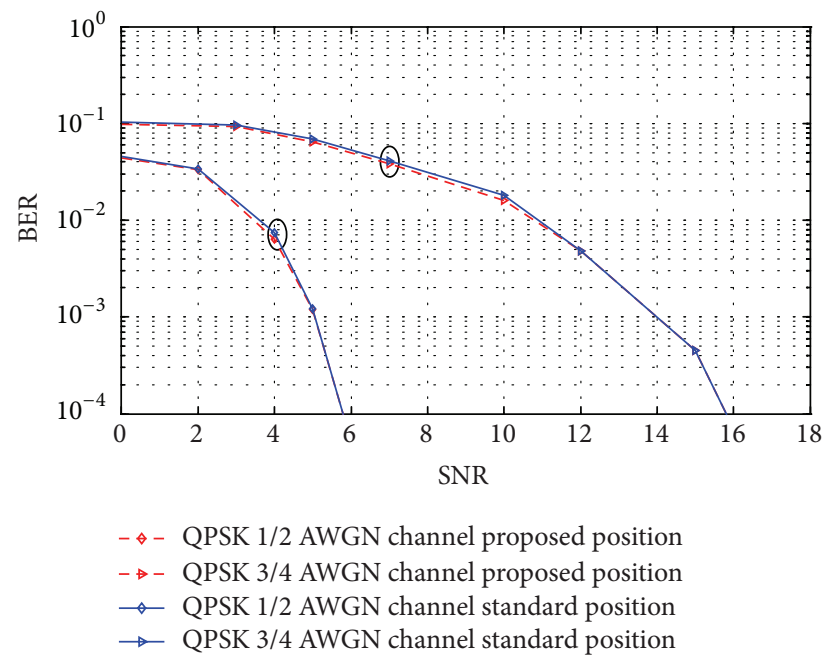

FIGURE 15: Standardized estimation versus new pilot position estimation for QPSK.

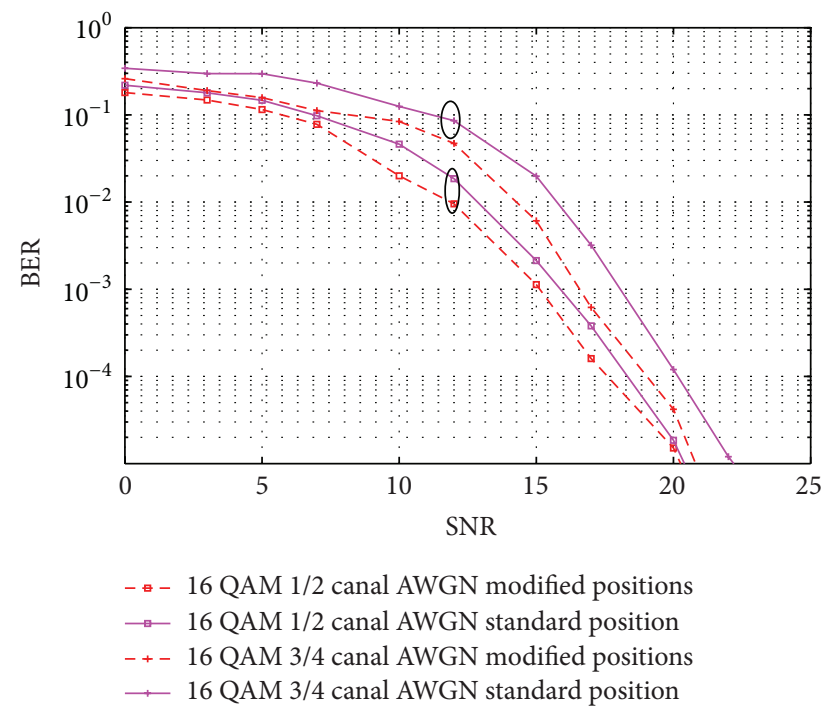

FIGURE 16: Standardized estimation versus new pilot position estimation for 16 QAM.

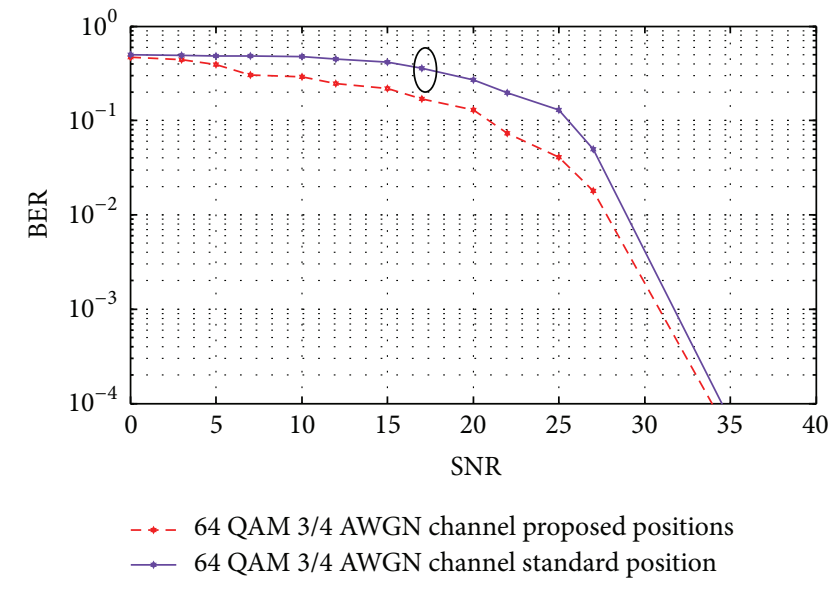

FIGURE 17: Standardized estimation versus new pilot position estimation for 64 QAM 2/3.

worsens with the increase in the node speed. In fact, for BPSK $1 / 2$ modulation, when the node speed increases from 20 to $220 \mathrm{~km} / \mathrm{h}$, the PLR degrades from $0 \%$ to $18 \%$, respectively. The main contribution of this research work is that communications at high data rate are needed in potential applications of the studied system and that extra effort is needed to ameliorate the performance of communications taken at high mobility.

Generally, the obtained results from the simulation and real-world experimentation indicate that the implemented model helped to achieve a relatively good description of the real communication involved in our intervehicular communication. Taking the promising results and conclusions yielded by our model into account, we have chosen the pilot based estimation to ameliorate the V2X communication performances as much as possible. In a first step, the results offered by the rearranging pilot subcarriers were proven to be better than those provided by the standardized position. Then, in a second step, the addition of two new pilot subcarriers was confirmed to improve the V2X communication performance. It has also been proven that attaching more pilot subcarriers may have an effect on the spectral efficiency with the growth of SEF from 0.076 to 0.115 . In short, although 


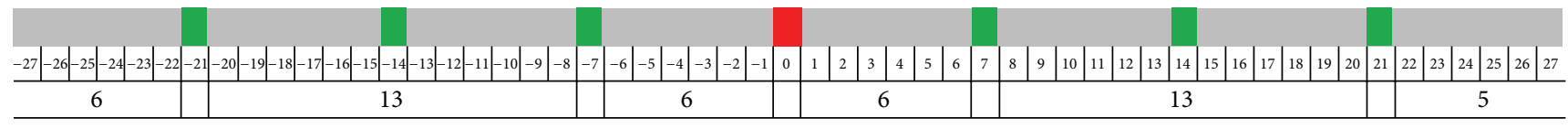

FIGURE 18: Transmission media with two added subcarriers.

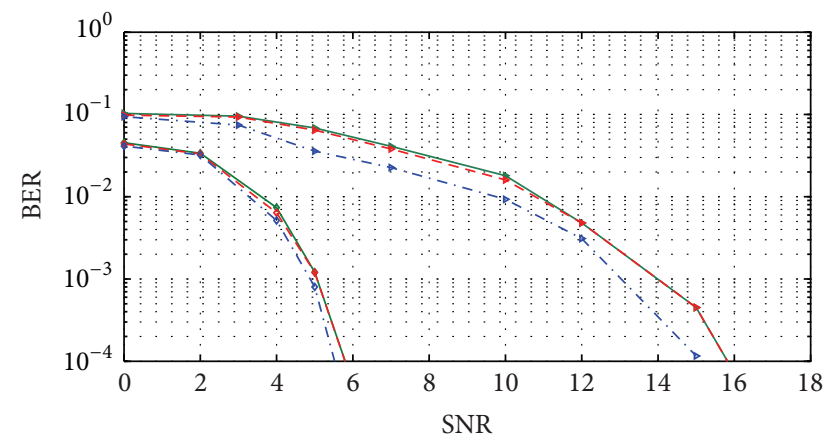

$\rightarrow$ QPSK 1/2 AWGN channel standard position

- - QPSK 1/2 AWGN channel modified positions

$\rightarrow \rightarrow$ QPSK 1/2 AWGN channel 6 pilots

$\rightarrow$ QPSK 3/4 AWGN standard position

$\rightarrow-$ QPSK 3/4 AWGN channel modified positions

$\rightarrow \rightarrow$ QPSK 3/4 AWGN channel 6 pilots

FIGURE 19: Comparison between the added pilot subcarrier and repositioned pilot subcarrier for QPSK modulation.

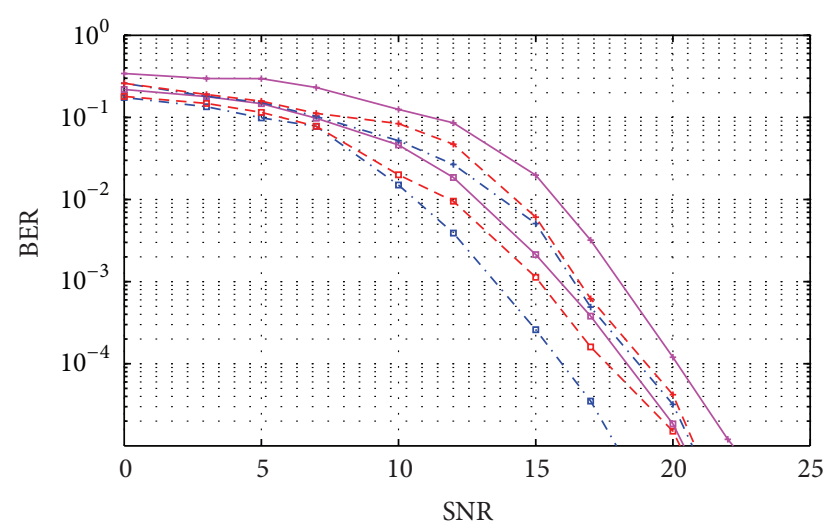

- - 16 QAM 1/2 channel AWGN with 6 pilots

- -16 QAM 1/2 channel AWGN modified positions

$\simeq 16 \mathrm{QAM} 1 / 2$ channel AWGN standard position

.+ - 16 QAM 3/4 channel de AWGN with 6 pilots

- +- 16 QAM 3/4 channel AWGN modified positions

$\longrightarrow 16$ QAM 3/4 channel AWGN standard

FIGURE 20: Comparison between the added pilot subcarrier and repositioned pilot subcarrier for 16 QAM modulation.

the suggested approach may be an improvement solution, a spectral problem still remains to be outdone.

\section{Competing Interests}

The authors declare that they have no competing interests.

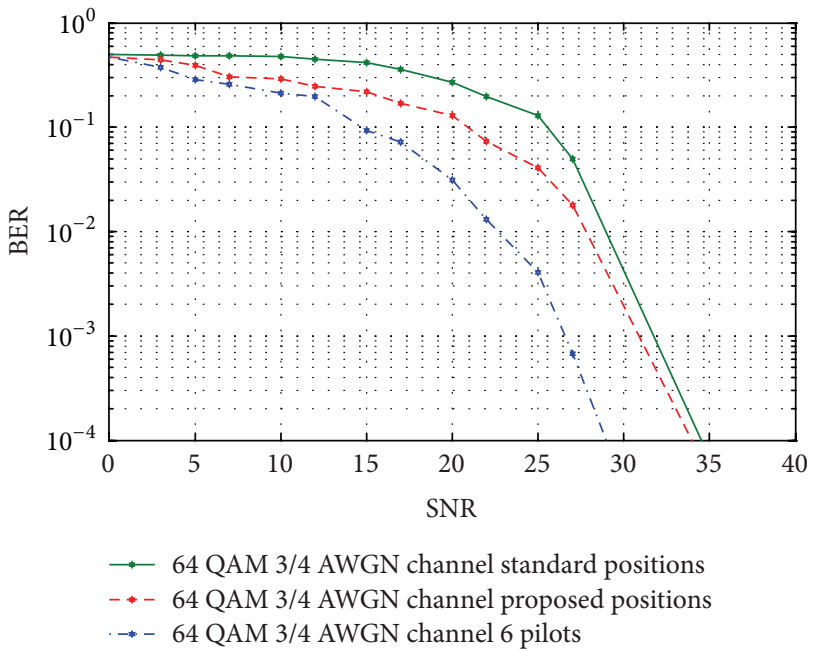

FIgure 21: Comparison between the added pilot subcarrier and repositioned pilot subcarrier for 64 QAM modulation.

\section{References}

[1] S. Grant-Muller and M. Usher, "Intelligent transport systems: the propensity for environmental and economic benefits," Technological Forecasting and Social Change, vol. 82, no. 1, pp. 149166, 2014.

[2] A. Festag, "Cooperative intelligent transport systems standards in Europe," IEEE Communications Magazine, vol. 52, no. 12, pp. 166-172, 2014.

[3] R. Bishop, "Intelligent vehicle applications worldwide," IEEE Intelligent Systems and their Applications, vol. 15, no. 1, pp. 78-81, 2002.

[4] F. Dressler, H. Hartenstein, O. Altintas, and O. K. Tonguz, "Inter-vehicle communication: quo vadis," IEEE Communications Magazine, vol. 52, no. 6, pp. 170-177, 2014.

[5] C. Diakaki, M. Papageorgiou, I. Papamichail, and I. Nikolos, "Overview and analysis of Vehicle automation and communication systems from a motorway traffic management perspective," Transportation Research Part A: Policy and Practice, vol. 75, pp. 147-165, 2015.

[6] G. Maier, A. Paier, and C. F. Mecklenbrauker, "Performance evaluation of IEEE 802.11p infrastructure-to-vehicle real-world measurements with receive diversity," in Proceedings of the 8th IEEE International Wireless Communications and Mobile Computing Conference (IWCMC '12), pp. 1113-1118, Limassol, Cyprus, August 2012.

[7] K. Ashokkumar, S. Baron, R. Arshadprabhu, and Britto, "Cloud based intelligent transport system," Procedia Computer Science Journal, vol. 50, pp. 58-63, 2015.

[8] B. Boyaci, K. G. Zografos, and N. Geroliminis, "An optimization framework for the development of efficient one-way car-sharing systems," European Journal of Operational Research, vol. 240, no. 3, 2015. 
[9] S. Eichler, "Performance evaluation of the IEEE 802.11p WAVE communication standard," in Proceedings of the IEEE 66th Vehicular Technology Conference (VTC '07), pp. 2199-2203, Baltimore, Md, USA, October 2007.

[10] Y. Wang, A. Ahmed, B. Krishnamachari, and K. Psounis, "IEEE 802.11p performance evaluation and protocol enhancement," in Proceedings of the IEEE International Conference on Vehicular Electronics and Safety (ICVES '08), pp. 317-322, Columbus, Ohio, USA, September 2008.

[11] A. Jafari, S. Al-Khayatt, and A. Dogman, "Performance evaluation of IEEE 802.11p for vehicular communication networks," in Proceedings of the 8th International Symposium on Communication Systems, Networks \& Digital Signal Processing (CSNDSP '12), pp. 1-5, IEEE, Poznań, Poland, July 2012.

[12] C.-K. Park, K.-H. Cho, M.-W. Ryu, and S.-H. Cha, "Measuring the performance of packet size and data rate for vehicular ad hoc networks," in Proceedings of the 4th International Conference on Information Science and Applications (ICISA '13), pp. 1-2, IEEE, Suwon, South Korea, June 2013.

[13] A. Sassi, F. Charfi, Y. Elhillali, L. Kamun, and A. Mrivenq, "PHY layer performance evaluation of the IEEE 802.11p through real-world experiments," in Proceedings of the 5th International Conference on Industrial Engeneering and Systems Management, October 2013.

[14] S. Demmel, A. Lambert, D. Gruyer, A. Rakotonirainy, and E. Monacelli, "Empirical IEEE 802.11p performance evaluation on test tracks," in Proceedings of the IEEE Intelligent Vehicles Symposium (IV '12), pp. 837-842, June 2012.

[15] J. Gozalvez, M. Sepulcre, and R. Bauza, "IEEE 802.11p vehicle to infrastructure communications in urban environments," IEEE Communications Magazine, vol. 50, no. 5, pp. 176-183, 2012.

[16] A. Sassi, F. Charfi, Y. Elhillali, L. Kamun, and A. MRivenq, "OFDM transmission performance evaluation in V2X communication," IJCSI International Journal of Computer Science, vol. 9, no. 2, pp. 141-148, 2012.

[17] A. Paier, R. Tresch, A. Alonso et al., "Average downstream performance of measured IEEE 802.11p infrastructure-to-vehicle links," in Proceedings of the IEEE International Conference on Communications Workshops (ICC '10), Capetown, South Africa, May 2010.

[18] A. Paier, D. Faetani, and C. F. Mecklenbräuker, "Performance evaluation of IEEE 802.11p physical layer infrastructure-tovehicle real-world measurements," in Proceedings of the $3 \mathrm{rd}$ International Symposium on Applied Sciences in Biomedical and Communication Technologies (ISABEL '10), pp. 1-5, IEEE, Rome, Italy, November 2010.

[19] T. M. Fernández-Caramés, M. González-López, C. J. Escudero, and L. Castedo, "Performance evaluation of multiple-antenna IEEE 802.11p transceivers using an FPGA-based MIMO vehicular channel emulator," EURASIP Journal on Wireless Communications and Networking, vol. 2012, article 215, 2012.

[20] C.-S. Lin, C.-K. Sun, J.-C. Lin, and B.-C. Chen, "Performance evaluations of channel estimations in IEEE 802.11p environments," in Proceedings of the International Conference on Ultra Modern Telecommunications \& Workshops, pp. 1-5, IEEE, St. Petersburg, Russia, October 2009.

[21] Z. Zhao, X. Cheng, M. Wen, B. Jiao, and C.-X. Wang, "Channel estimation schemes for IEEE 802.11p standard," IEEE Intelligent Transportation Systems Magazine, vol. 5, no. 4, pp. 38-49, 2013.

[22] T. Sukuvaara, R. Ylitalo, and M. Katz, "IEEE 802.11p based vehicular networking operational pilot field measurement," IEEE
Journal on Selected Areas in Communications, vol. 31, no. 9, pp. 409-417, 2013.

[23] http://www.safespot-eu.org/consortium.html.

[24] http://www.drive-c2x.eu/project.

[25] https://project.inria.fr/scoref/.

[26] http://www.simtd.de/index.dhtml/deDE/index.html.

[27] http://www.ict-itetris.eu/.

[28] http://www.wisafecar.com/.

[29] Draft amendment to standard for information technology telecommunications and information exchange between systems local and metropolitan area networks specific requirements Part 11: wireless LAN medium access control(MAC) and physical layer specifications Amendment 7: wireless access in vehicular environment, 2007.

[30] IEEE, "Part 11: wireless LAN Medium Access Control (MAC) and Physical Layer (PHY) specifications high-speed physical layer in the $5 \mathrm{GHz}$ band," IEEE Std 802.11-2007, 1999. 


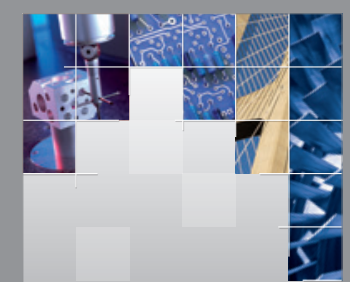

\section{Enfincering}
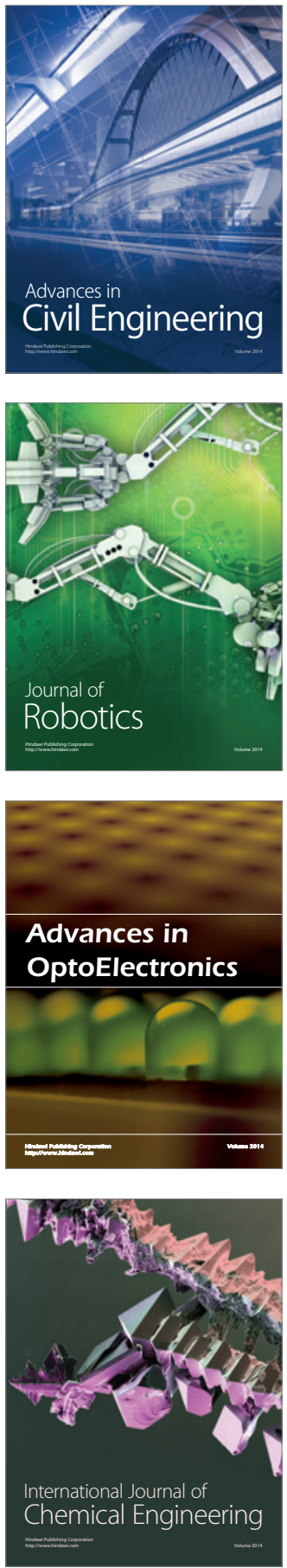

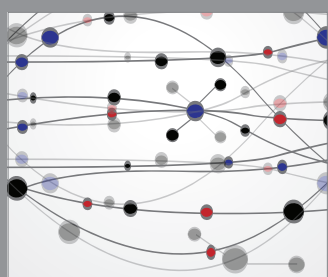

The Scientific World Journal

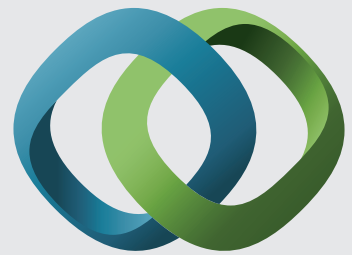

\section{Hindawi}

Submit your manuscripts at

http://www.hindawi.com
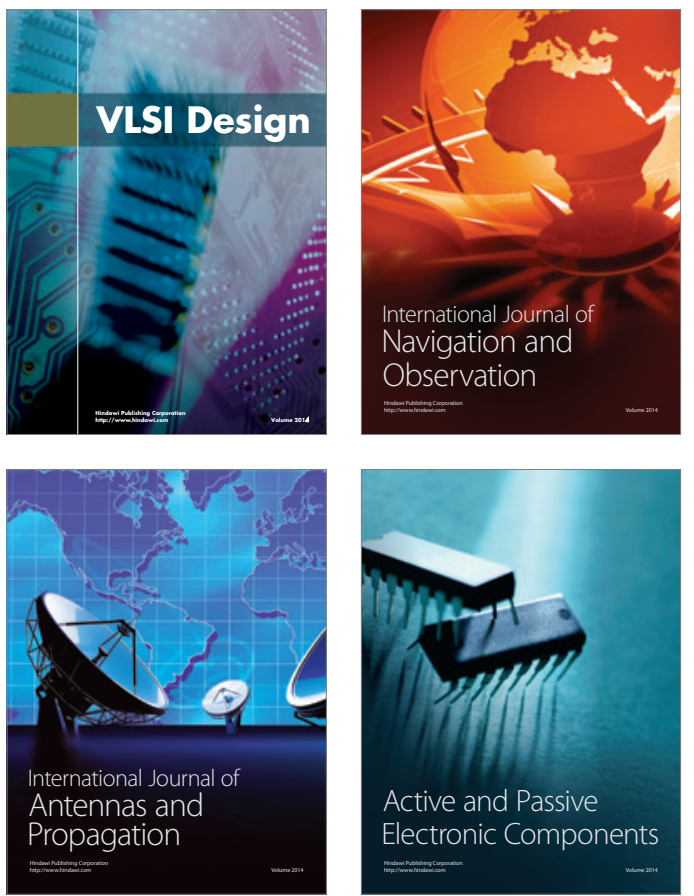
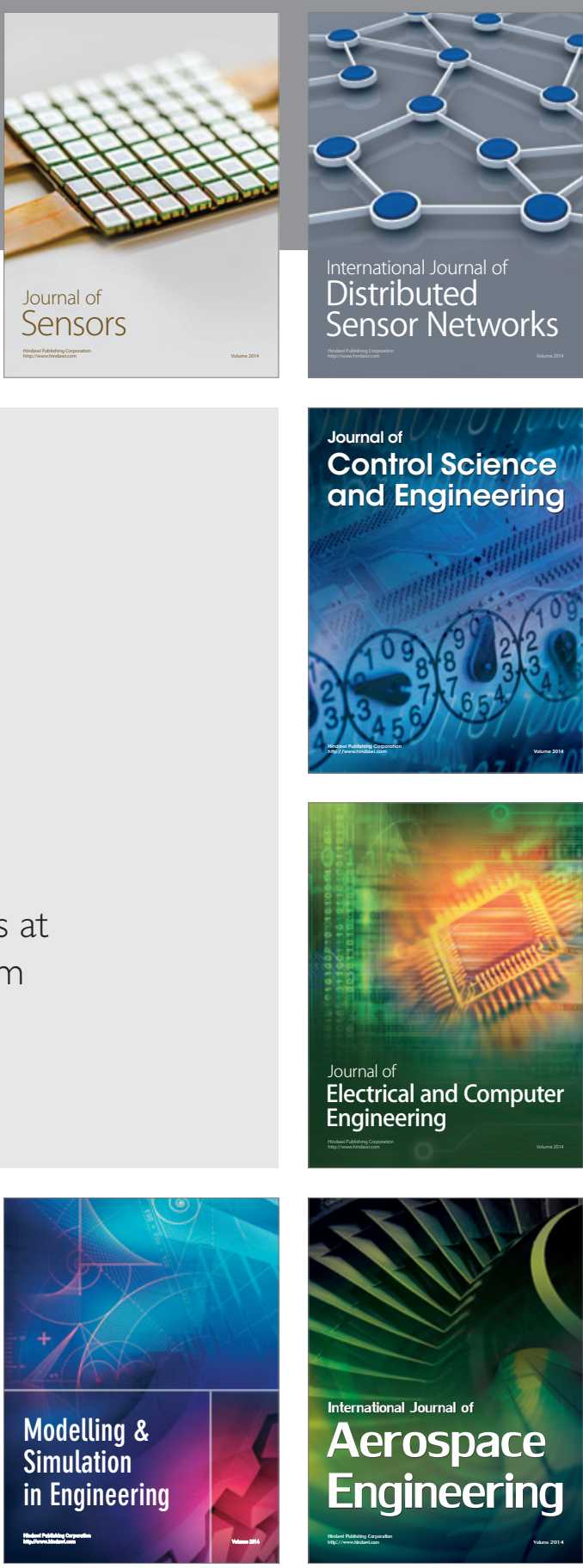

International Journal of

Distributed

Sensor Networks

Journal of

Control Science

and Engineering
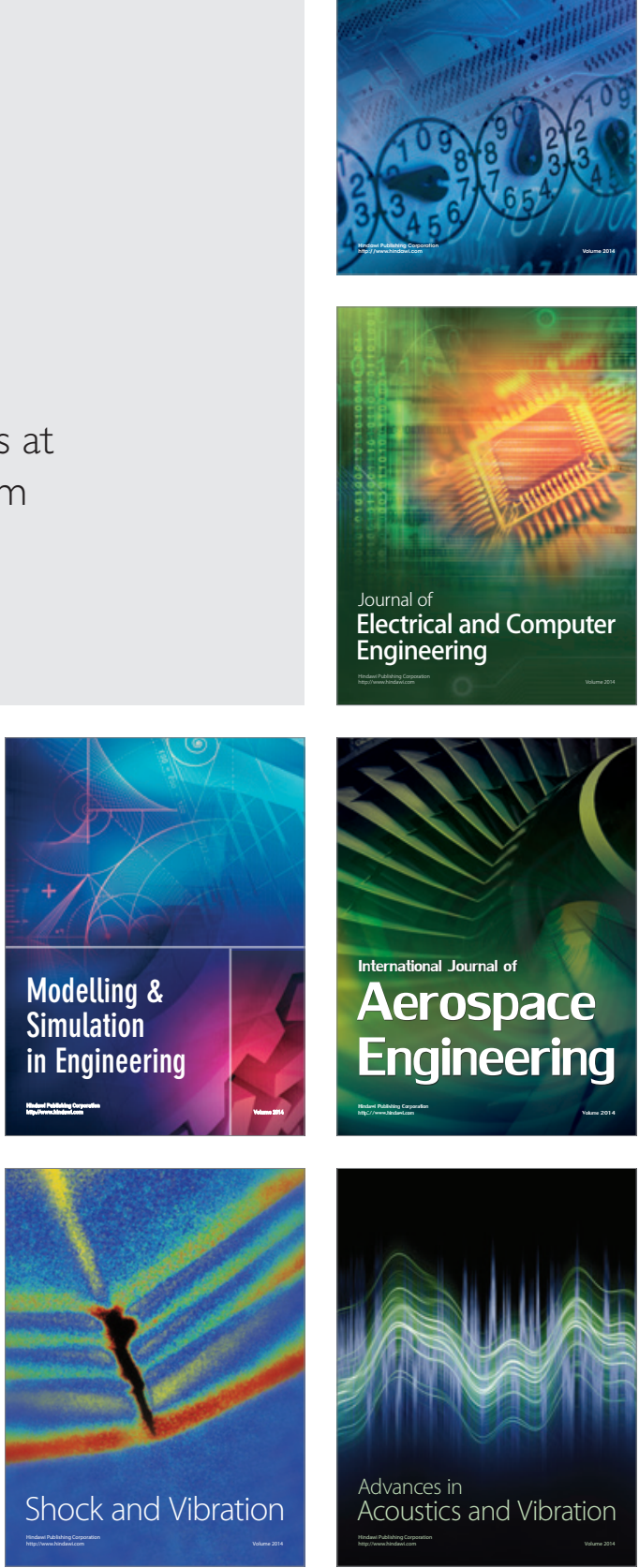